\title{
Singularities and Thermodynamics of Geodesic Surface Congruences
}

\author{
Yong Seung Cho and Soon-Tae Hong \\ Ewha Womans University \\ Korea
}

\section{Introduction}

In this chapter, we study the singularity of the geodesic surface congruence for time-like and null strings using the expansion of the universe in the string theory. We have Raychaudhuri type equation for the expansion. Assuming the stringy strong energy condition and initial convergence, we induce the existence of a Hawking-Penrose type singularity and obtain the same inequality equation of the stringy strong energy condition for timelike and null stringy congruences.

We consider the variation of the surface spanned by closed strings in a space-time manifold. Using the Nambu-Goto string action, we induce the geodesic surface equation and the geodesic surface deviation equation which yields a Jacobi field, and we define the index form of a geodesic surface as in the case of point particles to discuss conjugate strings on the geodesic surface.

Using the Nambu-Goto string action in the space of the surfaces spanned by closed strings in a spacetime manifold, we investigated the geodesic surface equation in the space of surfaces joining two given strings and the geodesic surface deviation equation in geodesic surface congruence which yields a Jacobi field along a given geodesic surface, and singularities in geodesic surface congruences. Assuming that the singularity exists in geodesic surface congruences in a conformally symmetric manifold, we compute the Jacobi fields.

In the standard cosmology, the universe expanded from a single point, namely Big Bang, and then a radiation dominated phase occurred followed by a matter dominated one, together with a phase transition between these two phases. Stringy cosmology displays features that are different from standard cosmology. One may be surprised in that in the stringy scenario there is no phase transition between the radiation and matter dominated phases, and the universe repeats a cyclic pattern in its evolution similar to brane cyclic cosmology. The Large Hadron Collider at CERN is expected to be able to determine which cosmology among several scenarios including the stringy cosmology is viable through detecting the quark-gluon plasma state and its ensuing evolution of the tiny universe. This chapter is mainly based on the articles Cho \& Hong (2007; 2008); Cho (2010); Cho \& Hong (2010).

\section{Morse theory in the space of surfaces}

\subsection{Stringy geodesic surfaces in Morse theory}

In analogy of the relativistic action of a point particle, the Nambu-Goto action Goto (1971); Nambu (1970) for a closed string is proportional to the area of the surface spanned in 
spacetime manifold $M$ by the evolution of the string. In order to define the action on the curved manifold, let $\left(M, g_{a b}\right)$ be an $n$-dimensional manifold associated with the metric $g_{a b}$. Given $g_{a b}$, we can have a unique covariant derivative $\nabla_{a}$ satisfying Wald (1984) $\nabla_{a} g_{b c}=0$, $\nabla_{a} \omega^{b}=\partial_{a} \omega^{b}+\Gamma_{a c}^{b} \omega^{c}$ and

$$
\left(\nabla_{a} \nabla_{b}-\nabla_{b} \nabla_{a}\right) \omega_{c}=R_{a b c}^{d} \omega_{d}
$$

We parameterize the surface $\gamma(\tau, \sigma)$ spanned by a closed string by two internal coordinates $\sigma$ and $\tau$, and then we have the corresponding vector fields $\xi^{a}=(\partial / \partial \tau)^{a}$ and $\zeta^{a}=(\partial / \partial \sigma)^{a}$. The Nambu-Goto string action is then given by Goto (1971); Nambu (1970)

$$
S=-\int_{0}^{T} \int_{0}^{2 \pi} d \tau d \sigma f(\tau, \sigma)
$$

where

$$
f(\tau, \sigma)=\left[(\xi \cdot \zeta)^{2}-(\xi \cdot \xi)(\zeta \cdot \zeta)\right]^{1 / 2}
$$

We now perform an infinitesimal variation of the tubes $\gamma_{\alpha}(\tau, \sigma)$ traced by the closed string during its evolution in order to find the geodesic surface equation from the least action principle. Here we impose the restriction that the length of the string circumference is $\tau$ independent for simplicity. Let the vector field $\eta^{a}=(\partial / \partial \alpha)^{a}$ be the deviation vector which represents the displacement to an infinitesimally nearby tube, and let $\Sigma$ denote the three-dimensional submanifold spanned by the tubes $\gamma_{\alpha}(\tau, \sigma)$. We then may choose $\tau, \sigma$ and $\alpha$ as coordinates of $\Sigma$ to yield the commutator relations,

$$
\begin{aligned}
& £_{\xi} \eta^{a}=\xi^{b} \nabla_{b} \eta^{a}-\eta^{b} \nabla_{b} \xi^{a}=0, \\
& £_{\zeta} \eta^{a}=\zeta^{b} \nabla_{b} \eta^{a}-\eta^{b} \nabla_{b} \zeta^{a}=0, \\
& £_{\xi} \zeta^{a}=\xi^{b} \nabla_{b} \zeta^{a}-\zeta^{b} \nabla_{b} \xi^{a}=0 .
\end{aligned}
$$

Now we find the first variation as follows

$$
\begin{aligned}
\frac{d S}{d \alpha}= & -\iint d \tau d \sigma \eta^{a} \nabla_{a} f \\
= & -\iint d \tau d \sigma\left(P_{\tau}^{b} \xi^{a} \nabla_{a} \eta_{b}+P_{\sigma}^{b} \zeta^{a} \nabla_{a} \eta_{b}\right) \\
= & \iint d \tau d \sigma \eta_{b}\left(\xi^{a} \nabla_{a} P_{\tau}^{b}+\zeta^{a} \nabla_{a} P_{\sigma}^{b}\right) \\
& -\left.\int d \sigma P_{\tau}^{b} \eta_{b}\right|_{\tau=0} ^{\tau=T}-\left.\int d \tau P_{\sigma}^{b} \eta_{b}\right|_{\sigma=0} ^{\sigma=2 \pi}
\end{aligned}
$$

where

$$
\begin{aligned}
& P_{\tau}^{a}=\frac{1}{f}\left[(\xi \cdot \zeta) \zeta^{a}-(\zeta \cdot \zeta) \xi^{a}\right], \\
& P_{\sigma}^{a}=\frac{1}{f}\left[(\xi \cdot \zeta) \xi^{a}-(\xi \cdot \xi) \zeta^{a}\right]
\end{aligned}
$$


are the energy-momentum currents. Using the endpoint conditions $\eta^{a}(0)=\eta^{a}(T)=0$ and periodic condition $\eta^{a}(\sigma+2 \pi)=\eta^{a}(\sigma)$, we have the geodesic surface equation

$$
\xi^{a} \nabla_{a} P_{\tau}^{b}+\zeta^{a} \nabla_{a} P_{\sigma}^{b}=0,
$$

and the constraint identities

$$
\begin{aligned}
& P_{\tau} \cdot \zeta=0, P_{\tau} \cdot P_{\tau}+\zeta \cdot \zeta=0, \\
& P_{\sigma} \cdot \xi=0, P_{\sigma} \cdot P_{\sigma}+\xi \cdot \xi=0 .
\end{aligned}
$$

Let $\gamma_{\alpha}(\tau, \sigma)$ denote a smooth one-parameter family of geodesic surfaces: for each $\alpha \in \mathbf{R}$, the tube $\gamma_{\alpha}$ is a geodesic surface parameterized by affine parameters $\tau$ and $\sigma$. For an infinitesimally nearby geodesic surface in the family, we then have the following geodesic surface deviation equation

$$
\xi^{b} \nabla_{b}\left(\eta^{c} \nabla_{c} P_{\tau}^{a}\right)+\zeta^{b} \nabla_{b}\left(\eta^{c} \nabla_{c} P_{\sigma}^{a}\right)+R_{b c d}^{a}\left(\xi^{b} P_{\tau}^{d}+\zeta^{b} P_{\sigma}^{d}\right) \eta^{c} \equiv(\Lambda \eta)^{a}=0 .
$$

For an infinitesimal $\alpha$, our goal is to investigate the variation vector field $\eta^{a}$ by comparing $S(0)$ with $S(\alpha)$ with $S(0)$ along the time direction $\tau$. The second variation $d^{2} S / d \alpha^{2}(0)$ is then needed only when $d S / d \alpha(0)=0$. Explicitly, the second variation is given by

$$
\begin{aligned}
\left.\frac{d^{2} S}{d \alpha^{2}}\right|_{\alpha=0}= & -\iint d \tau d \sigma \eta^{b} \nabla_{b}\left(\eta^{a} \nabla_{a} f\right) \\
= & -\iint d \tau d \sigma\left[\left(\eta^{c} \nabla_{c} P_{\tau}^{b}\right)\left(\xi^{a} \nabla_{a} \eta_{b}\right)+\left(\eta^{c} \nabla_{c} P_{\sigma}^{b}\right)\left(\zeta^{a} \nabla_{a} \eta_{b}\right)\right. \\
& \left.-R_{a c b}{ }^{d}\left(\xi^{a} P_{\tau}^{b}+\zeta^{a} P_{\sigma}^{b}\right) \eta^{c} \eta_{d}\right] \\
& -\left.\int d \sigma P_{\tau}^{b} \eta^{a} \nabla_{a} \eta_{b}\right|_{\tau=0} ^{\tau=T}-\left.\int d \tau P_{\sigma}^{b} \eta^{a} \nabla_{a} \eta_{b}\right|_{\sigma=0} ^{\sigma=2 \pi} .
\end{aligned}
$$

Here the boundary terms vanish for the fixed endpoint and the periodic conditions, even though on the geodesic surface we have breaks which we will explain later. After some algebra using the geodesic surface deviation equation, we have

$$
\left.\frac{d^{2} S}{d \alpha^{2}}\right|_{\alpha=0}=\iint d \tau d \sigma \eta_{a}(\Lambda \eta)^{a}
$$

\subsection{Jacobi fields in orthonormal gauge}

The string action and the corresponding equations of motion are invariant under reparameterization $\tilde{\sigma}=\tilde{\sigma}(\tau, \sigma)$ and $\tilde{\tau}=\tilde{\tau}(\tau, \sigma)$. We have then gauge degrees of freedom so that we can choose the orthonormal gauge as follows

$$
\xi \cdot \zeta=0, \quad \xi \cdot \xi+\zeta \cdot \zeta=0,
$$

where the plus sign in the second equation is due to the fact that $\xi \cdot \xi$ is timelike and $\zeta \cdot \zeta$ is spacelike. In this parameterization the energy-momentum currents (8) satisfying the constraints (10) are

$$
P_{\tau}^{a}=-\xi^{a}, \quad P_{\sigma}^{a}=\zeta^{a}
$$


The geodesic surface equation and the geodesic surface deviation equation read

$$
\begin{aligned}
& -\xi^{a} \nabla_{a} \xi^{b}+\zeta^{a} \nabla_{a} \zeta^{b}=0, \\
& -\xi^{b} \nabla_{b}\left(\xi^{c} \nabla_{c} \eta^{a}\right)+\zeta^{b} \nabla_{b}\left(\zeta^{c} \nabla_{c} \eta^{a}\right)-R_{b c d}^{a}\left(\xi^{b} \xi^{d}-\zeta^{b} \zeta^{d}\right) \eta^{c}=(\Lambda \eta)^{a}=0 .
\end{aligned}
$$

We now restrict ourselves to strings on constant scalar curvature manifold such as $S^{n}$. We take an ansatz that on this manifold the string shape on the geodesic surface $\gamma_{0}$ is the same as that on a nearby geodesic surface $\gamma_{\alpha}$ at a given time $\tau$. We can thus construct the variation vectors $\eta^{a}(\tau)$ as vectors associated with the centers of the string of the two nearby geodesic surfaces at the given time $\tau$. We then introduce an orthonormal basis of spatial vectors $e_{i}^{a}(i=$ $1,2, \ldots, n-2)$ orthogonal to $\xi^{a}$ and $\zeta^{a}$ and parallelly propagated along the geodesic surface. The geodesic surface deviation equation then yields for $(i, j=1,2, \ldots, n-2)$

$$
\frac{d^{2} \eta^{i}}{d \tau^{2}}+\left(R_{\tau j \tau}^{i}-R_{\sigma j \sigma}^{i}\right) \eta^{j}=0
$$

The value of $\eta^{i}$ at time $\tau$ must depend linearly on the initial data $\eta^{i}(0)$ and $\frac{d \eta^{i}}{d \tau}(0)$ at $\tau=0$. Since by construction $\eta^{i}(0)=0$ for the family of geodesic surfaces we must have

$$
\eta^{i}(\tau)=A_{j}^{i}(\tau) \frac{d \eta^{j}}{d \tau}(0)
$$

Inserting (18) into (17) we have the differential equation for $A_{j}^{i}(\tau)$

$$
\frac{d^{2} A_{j}^{i}}{d \tau^{2}}+\left(R_{\tau k \tau}^{i}-R_{\sigma k \sigma}^{i}\right) A_{j}^{k}=0,
$$

with the initial conditions

$$
A_{j}^{i}(0)=0, \quad \frac{d A_{j}^{i}}{d \tau}(0)=\delta_{j}^{i} .
$$

Note that in (19) we have the last term from the contribution of string property.

Next we consider the second variation equation (12) under the above restrictions

$$
\left.\frac{d^{2} S}{d \alpha^{2}}\right|_{\alpha=0}=\iint d \tau d \sigma\left(\frac{d \eta^{i}}{d \tau} \frac{\eta_{i}}{d \tau}-\left(R_{\tau j \tau}^{i}-R_{\sigma j \sigma}^{i}\right) \eta^{j} \eta_{i}\right) .
$$

Define the index form $I_{\gamma}$ of a geodesic surface $\gamma$ as the unique symmetric bilinear form $I_{\gamma}$ : $T_{\gamma} \times T_{\gamma} \rightarrow \mathbf{R}$ such that

$$
I_{\gamma}(V, V)=\left.\frac{d^{2} S}{d \alpha^{2}}\right|_{\alpha=0}(V, V)
$$

for $V \in T_{\gamma}$. From (21) we can easily find

$$
I_{\gamma}(V, W)=\iint d \tau d \sigma\left(\frac{d W^{m}}{d \tau} \frac{d V_{m}}{d \tau}-\left(R_{\tau j \tau}^{m}-R_{\sigma j \sigma}^{m}\right) W^{j} V_{m}\right) .
$$


If we have breaks $0=\tau_{0}<\cdots<\tau_{k+1}=T$, and the restriction of $\gamma$ to each set $\left[\tau_{i-1}, \tau_{i}\right]$ is smooth, then the tube $\gamma$ is piecewise smooth. The variation vector field $V$ of $\gamma$ is always piecewise smooth. However $d V / d \tau$ will generally have a discontinuity at each break $\tau_{i}(1 \leq$ $i \leq k)$. This continuity is measured by

$$
\Delta \frac{d V}{d \tau}\left(\tau_{i}\right)=\frac{d V}{d \tau}\left(\tau_{i}^{+}\right)-\frac{d V}{d \tau}\left(\tau_{i}^{-}\right),
$$

where the first term is derived from the restrictions $\gamma \mid\left[\tau_{i}, \tau_{i+1}\right]$ and the second from $\gamma \mid\left[\tau_{i-1}, \tau_{i}\right]$. If $\gamma$ and $V \in T_{\gamma}$ have the breaks $\tau_{1}<\cdots<\tau_{k}$ then except at breaks, we have

$$
\frac{d W^{m}}{d \tau} \frac{d V_{m}}{d \tau}=\frac{d}{d \tau}\left(V_{m} \frac{d W^{m}}{d \tau}\right)-V_{m} \frac{d^{2} W^{m}}{d \tau^{2}}
$$

and

$$
\sum_{i=0}^{k} \int_{\tau_{i}}^{\tau_{i+1}} \frac{d}{d \tau}\left(V_{m} \frac{d W^{m}}{d \tau}\right) d \tau=\left.\sum_{i=0}^{k} V_{m} \frac{d W^{m}}{d \tau}\right|_{\tau_{i}} ^{\tau_{i+1}}=-\sum_{i=0}^{k} V_{m} \Delta \frac{d W^{m}}{d \tau}\left(\tau_{i}\right)
$$

to yield

$$
\begin{aligned}
I_{\gamma}(V, W)= & -\iint d \tau d \sigma V^{m}\left(\frac{d^{2} W^{m}}{d \tau^{2}}+\left(R_{\tau j \tau}^{m}-R_{\sigma j \sigma}^{m}\right) W^{j}\right) \\
& -\sum_{i=0}^{k} \int d \sigma V_{m} \Delta \frac{d W^{m}}{d \tau}\left(\tau_{i}\right) .
\end{aligned}
$$

Here note that if we do not have the breaks, (21) yields

$$
\left.\frac{d^{2} S}{d \alpha^{2}}\right|_{\alpha=0}=-\iint d \tau d \sigma \eta_{i}\left(\frac{d^{2} \eta^{i}}{d \tau^{2}}+\left(R_{\tau j \tau}^{i}-R_{\sigma j \sigma}^{i}\right) \eta^{j}\right) .
$$

A solution $\eta^{a}$ of the geodesic surface deviation equation (17) is called a Jacobi field on the geodesic surface $\gamma$. A pair of points $p, q \in \gamma$ defined by the centers of the closed strings on the geodesic surface is then conjugate if there exists a Jacobi field $\eta^{a}$ which is not identically zero but vanishes at both $p$ and $q$. Roughly speaking, $p$ and $q$ are conjugate if an infinitesimally nearby geodesic surface intersects $\gamma$ at both $p$ and $q$. From (18), $q$ will be conjugate to $p$ if and only if there exists nontrivial initial data: $d \eta^{i} / d \tau(0) \neq 0$, for which $\eta^{i}=0$ at $q$. This occurs if and only if $\operatorname{det} A^{i}{ }_{j}=0$ at $q$, and thus $\operatorname{det} A^{i}{ }_{j}=0$ is the necessary and sufficient condition for a conjugate point to $p$. Note that between conjugate points, we have $\operatorname{det} A_{j}^{i} \neq 0$ and thus the inverse of $A_{j}^{i}$ exists. Using (19) we can easily see that

$$
\frac{d}{d \tau}\left(\frac{d A_{i j}}{d \tau} A_{k}^{i}-A_{i j} \frac{d A_{k}^{i}}{d \tau}\right)=0
$$


In addition, the quantity in parenthesis of (29) vanishes at $p$, since $A_{j}^{i}(0)=0$. Along a geodesic surface $\gamma$, we thus find

$$
\frac{d A_{i j}}{d \tau} A_{k}^{i}-A_{i j} \frac{d A_{k}^{i}}{d \tau}=0
$$

If $\gamma$ is a geodesic surface with no point conjugate to $p$ between $p$ and $q$, then $A^{i}{ }_{j}$ defined above will be nonsingular between $p$ and $q$. We can then define $Y^{i}=\left(A^{-1}\right)_{j}^{i} \eta^{j}$ or $\eta^{i}=A_{j}^{i} Y^{j}$. From (28) and (30), we can easily verify

$$
\left.\frac{d^{2} S}{d \alpha^{2}}\right|_{\alpha=0}=\iint d \tau d \sigma\left(A_{i j} \frac{d Y^{j}}{d \tau}\right)^{2} \geq 0 .
$$

Locally $\gamma$ minimizes the surface of the string, if $\gamma$ is a geodesic surface with no point conjugate to $p$ between $p$ and $q$.

On the other hand, if $\gamma$ is a geodesic surface but has a conjugate point $r$ between $p$ and $q$, then we have the Jacobi field $J^{i}$ along $\gamma$ which vanishes at $p$ and $r$. Extend $J^{i}$ to $q$ by putting it zero in $[r, q]$. Then $d J^{i} / d \tau\left(r^{-}\right) \neq 0$, since $J^{i}$ is nonzero. But $d J^{i} / d \tau\left(r^{+}\right)=0$ to yield

$$
\Delta \frac{d J^{i}}{d \tau}(r)=-\frac{d J^{i}}{d \tau}\left(r^{-}\right) \neq 0
$$

Choose any $k^{i} \in T_{\gamma}$ such that

$$
k_{i} \Delta \frac{d J^{i}}{d \tau}(r)=c,
$$

with positive constant $c$. Let $\eta^{i}$ be $\eta^{i}=\epsilon k^{i}+\epsilon^{-1} J^{i}$ where $\epsilon$ is some constant, then we have

$$
I_{\gamma}(\eta, \eta)=\epsilon^{2} I_{\gamma}(k, k)+2 I_{\gamma}(k, J)+\epsilon^{-2} I_{\gamma}(J, J) .
$$

By taking $\epsilon$ small enough, the first term in (34) vanishes and the third term also vanishes due to the definition of the Jacobi field. Using (33) we have $I_{\gamma}(k, J)=-2 \pi c$ and thus

$$
\left.\frac{d^{2} S}{d \alpha^{2}}\right|_{\alpha=0}=-4 \pi c
$$

which is negative definite. From the above arguments, we conclude that given a smooth timelike curve $\gamma$ connecting two points $p, q \in M$, the necessary and sufficient condition that $\gamma$ locally minimizes the surface of the closed string between $p$ and $q$ over smooth one parameter variations is that $\gamma$ is a geodesic surface with no point conjugate to $p$ between $p$ and $q$. It is also interesting to see that on $S^{n}$ we have $n-1$ conjugate points as in the case of point particle. Moreover, on the manifold with the constant scalar curvature $R$, the geodesic surfaces have no conjugate points for $R<0$ or $R=0$, while conjugate points occur for $R>0$. 


\section{Singularities in geodesic surface congruence for the time-like direction}

\subsection{Introduction}

The Hawking-Penrose singularity Hawking \& Penrose (1970) is assumed to exist at the beginning of the universe. In the standard inflationary cosmology based on the Hawking-Penrose singularity theorem and inflationary scenario, the universe is believed to expand from the Big Bang. Assuming that the early universe was filled with a perfect fluid consisting of massive particles and/or massless particles and using the strong energy condition which was used to show the Hawking-Penrose singularity theorem, one could find equations of state for each particle.

In the inflationary standard cosmology, it is believed that, after the Big Bang explosion, radiation dominated phase occurred followed by matter dominated one, even though there was a hot thermalization period of radiation and matter immediately after the Big Bang. Moreover, a phase transition exists between massive particle and massless particle phases in the universe. The equation of state of the massive particle is different from that of the massless particle, and thus the massive particle phase is not the same as the massless particle one.

Applying the string theory Green et al. (1987); Polchinski (1999) to cosmology, we might investigate the expansion of the universe in terms of the Hawking-Penrose singularity in geodesic surface congruences for the time-like and null strings Cho \& Hong (2008; 2010). Taking an ansatz that the expansion of the stringy congruence is constant along the string coordinate direction, we derive the Raychaudhuri type equation, which is an evolution equation for the expansion, possessing correction terms associated with the stringy configurations. Assuming the stringy strong energy condition, we induce the Hawking-Penrose type inequality equation which produces the same inequality equation for both the time-like and null stringy congruences.

\subsection{Congruence of strings}

The action for a string is proportional to the area of the surface spanned in spacetime manifold $M$ by the evolution of the string. In order to define the action on the curved manifold, we let $\left(M, g_{a b}\right)$ be a $D$-dimensional manifold associated with the metric $g_{a b}$. Given $g_{a b}$, we can have a unique covariant derivative $\nabla_{a}$ satisfying (1).

Parameterize the surface generated by the evolution of a string by two world sheet coordinates $\tau$ and $\sigma$, and then we have the corresponding vector fields $\xi^{a}=(\partial / \partial \tau)^{a}$ and $\zeta^{a}=$ $(\partial / \partial \sigma)^{a}$. Since we have gauge degrees of freedom, we can choose the orthonormal gauge as follows Scherk (1975)

$$
\xi \cdot \zeta=0, \quad \xi \cdot \xi+\zeta \cdot \zeta=0,
$$

where the plus sign in the second equation is due to the fact that $\xi \cdot \xi=-1$ is timelike and $\zeta \cdot \zeta=1$ is spacelike. In the orthonormal gauge, we introduce tensor fields $B_{a b}$ and $\bar{B}_{a b}$ defined as

$$
B_{a b}=\nabla_{b} \xi_{a}, \quad \bar{B}_{a b}=\nabla_{b} \zeta_{a},
$$

which satisfy the following identities

$$
\begin{aligned}
B_{a b} \xi^{a}=0, \quad \bar{B}_{a b} \zeta^{a} & =0 \\
-B_{a b} \xi^{b}+\bar{B}_{a b} \zeta^{b} & =0 .
\end{aligned}
$$


Here in the last equation, we have used the geodesic surface equation

$$
-\xi^{a} \nabla_{a} \xi^{b}+\zeta^{a} \nabla_{a} \zeta^{b}=0
$$

In particular, the timelike curves of the strings are geodesic, then the geodesic surface equation holds.

Let the vector field $\eta^{a}=(\partial / \partial \alpha)^{a}$ be the deviation vector which represents the displacement to an infinitesimally nearby world sheet, and we let $\Sigma$ denote the three-dimensional submanifold spanned by the world sheets $\gamma_{\alpha}(\tau, \sigma)$. We then may choose $\tau, \sigma$ and $\alpha$ as coordinates of $\Sigma$ to yield the commutator relations (4)-(6). Using the above relations, we have

$$
\xi^{a} \nabla_{a} \eta^{b}-\zeta^{a} \nabla_{a} \eta^{b}=\left(B_{a}^{b}-\bar{B}_{a}^{b}\right) \eta^{a} .
$$

Next introduce the metrics $h_{a b}$ and $\bar{h}_{a b}$,

$$
h_{a b}=g_{a b}+\xi_{a} \xi_{b}, \quad \bar{h}_{a b}=g_{a b}-\zeta_{a} \zeta_{b},
$$

which satisfy

$$
\begin{array}{lll}
h_{a b} \xi^{a}=0, & h_{a b} \xi^{b}=0, & h_{a b} g^{b c} h_{c d}=h_{a d}, \\
\bar{h}_{a b} \zeta^{a}=0, & \bar{h}_{a b} \zeta^{b}=0, & \bar{h}_{a b} g^{b c} \bar{h}_{c d}=\bar{h}_{a d}, \\
h_{a b} h^{a b}=D-1, \bar{h}_{a b} \bar{h}^{a b}=D-1, & h_{a b} \bar{h}^{a b}=D-2 .
\end{array}
$$

Here note that $h_{a b}$ and $\bar{h}_{a b}$ are the metrics on the hypersurfaces orthogonal to $\xi^{a}$ and $\zeta^{a}$, respectively. Moreover, we can define projection operators $h^{a}{ }_{b}$ and $\bar{h}_{b}^{a}$ as follows

$$
h_{b}^{a}=g^{a c} h_{c b}, \quad \bar{h}_{b}^{a}=g^{a c} \bar{h}_{c b} .
$$

These operators fulfil

$$
\begin{gathered}
h_{b}^{a} h_{c}^{b}=h^{a b} h_{b c}=h^{a}, \bar{h}^{a} b_{h^{b}}{ }_{c}=\bar{h}^{a b} \bar{h}_{b c}=\bar{h}^{a}{ }_{c}, \\
h_{a b} h^{b c} h_{c d}=h_{a d}, \quad \bar{h}_{a b} \bar{h}^{b c} \bar{h}_{c d}=\bar{h}_{a d} .
\end{gathered}
$$

Now, decompose $B_{a b}$ into three pieces

$$
B_{a b}=\frac{1}{D-1} \theta h_{a b}+\sigma_{a b}+\omega_{a b}
$$

where the expansion $\theta$, the shear $\sigma_{a b}$ and the twist $\omega_{a b}$ of the stringy congruence are given by

$$
\theta=B^{a b} h_{a b}, \quad \sigma_{a b}=B_{(a b)}-\frac{1}{D-1} \theta h_{a b}, \quad \omega_{a b}=B_{[a b]} .
$$

Similarly, $\bar{B}_{a b}$ is also decomposed into three parts

$$
\bar{B}_{a b}=\frac{1}{D-1} \bar{\theta} \bar{h}_{a b}+\bar{\sigma}_{a b}+\bar{\omega}_{a b}
$$

where

$$
\bar{\theta}=\bar{B}^{a b} \bar{h}_{a b}, \quad \bar{\sigma}_{a b}=\bar{B}_{(a b)}-\frac{1}{D-1} \bar{\theta} \bar{h}_{a b}, \quad \bar{\omega}_{a b}=\bar{B}_{[a b]} .
$$


We then find

$$
\begin{array}{cc}
\sigma_{a b} h^{a b}=0, & \omega_{a b} h^{a b}=0, \\
\bar{\sigma}_{a b} \bar{h}^{a b}=0, & \bar{\omega}_{a b} \bar{h}^{a b}=0, \\
-\sigma_{a b} \xi^{b}+\bar{\sigma}_{a b} \zeta^{b}=0,-\omega_{a b} \xi^{b}+\bar{\omega}_{a b} \zeta^{b}=0,
\end{array}
$$

and

$$
-\xi^{c} \nabla_{c} B_{a b}+\zeta^{c} \nabla_{c} \bar{B}_{a b}=B_{b}^{c} B_{a c}-\bar{B}_{b}^{c} \bar{B}_{a c}-R_{c b a d}\left(\xi^{c} \xi^{d}-\zeta^{c} \zeta^{d}\right) .
$$

Exploiting (50) one arrives at

$$
\begin{aligned}
-\xi^{a} \nabla_{a} \theta+\zeta^{a} \nabla_{a} \bar{\theta}= & \frac{1}{D-1}\left(\theta^{2}-\bar{\theta}^{2}\right)+\sigma_{a b} \sigma^{a b}-\bar{\sigma}_{a b} \bar{\sigma}^{a b}-\omega_{a b} \omega^{a b}+\bar{\omega}_{a b} \bar{\omega}^{a b} \\
& +R_{a b}\left(\xi^{a} \xi^{b}-\zeta^{a} \zeta^{b}\right) .
\end{aligned}
$$

\subsection{Expansion of timelike stringy congruence}

The motion types of stringy congruence can be described in terms of expansion, twist and shear. In this section, we will pedagogically summarize the previous results Cho \& Hong $(2008 ; 2010)$ on the expansion rate of stringy congruence in the early universe for the sake of completeness. We will consider the twist and shear motions in the next section.

Taking an ansatz that the expansion $\bar{\theta}$ is constant along the $\sigma$-direction, from (51) one obtains a Raychaudhuri type equation

$$
\frac{d \theta}{d \tau}=-\frac{1}{D-1}\left(\theta^{2}-\bar{\theta}^{2}\right)-\sigma_{a b} \sigma^{a b}+\bar{\sigma}_{a b} \bar{\sigma}^{a b}+\omega_{a b} \omega^{a b}-\bar{\omega}_{a b} \bar{\omega}^{a b}-R_{a b}\left(\xi^{a} \xi^{b}-\zeta^{a} \zeta^{b}\right) .
$$

Assume that $\omega_{a b}=\bar{\omega}_{a b}, \sigma_{a b}=\bar{\sigma}_{a b}$ and a stringy strong energy condition

$$
R_{a b}\left(\xi^{a} \xi^{b}-\zeta^{a} \zeta^{b}\right)=8 \pi\left(T_{a b}\left(\xi^{a} \xi^{b}-\zeta^{a} \zeta^{b}\right)+\frac{2}{D-2} T\right) \geq 0,
$$

where $T_{a b}$ and $T$ are the energy-momentum tensor and its trace, respectively. The Raychaudhuri type equation (52) then has a solution of the form

$$
\frac{1}{\theta(\tau)} \geq \frac{1}{\theta(0)}+\frac{1}{D-1}\left(\tau-\int_{0}^{\tau} \mathrm{d} \tau\left(\frac{\bar{\theta}}{\bar{\theta}}\right)^{2}\right)
$$

Assume that $\theta(0)$ is negative so that the congruence is initially converging as in the point particle case. The inequality (54) implies that $\theta(\tau)$ must pass through the singularity within a proper time

$$
\tau \leq \frac{D-1}{|\theta(0)|}+\int_{0}^{\tau} \mathrm{d} \tau\left(\frac{\bar{\theta}}{\bar{\theta}}\right)^{2}
$$

For a perfect fluid, the energy-momentum tensor given by

$$
T_{a b}=\rho u_{a} u_{a}+P\left(g_{a b}+u_{a} u_{b}\right)
$$

where $\rho$ and $P$ are the mass-energy density and pressure of the fluid as measured in its rest frame, respectively, and $u^{a}$ is the time-like $D$-velocity in its rest frame Misner et al. (1972); 
Wald (1984), the stringy strong energy condition (53) yields only one inequality equation

$$
\frac{D-4}{D-2} \rho+\frac{D}{D-2} P \geq 0
$$

Now, we consider the point particle limit of the timelike stringy congruence. If the fiber space $F$ in the fibration $\pi: M \rightarrow N_{4}$ is a point, then the total space $M$ is the same as the base spacetime four manifold $N_{4}$. In this case, the geodesic surfaces are geodesic in $N_{4}$, the congruence of time-like geodesic surfaces is a congruence of time-like geodesics, and so $\bar{B}_{a b}=\bar{\theta}=\bar{\sigma}_{a b}=\bar{\omega}_{a b}=0$. If the congruence is hypersurface orthogonal, then we have $\omega_{a b}=0$. Suppose that the strong energy condition $R_{a b} \xi^{a} \xi^{b} \geq 0$ is satisfied to yield two inequalities Carroll (2004); Hawking \& Penrose (1970); Wald (1984)

$$
\rho+3 P \geq 0, \quad \rho+P \geq 0 .
$$

We then have the differential inequality equation

$$
\frac{d \theta}{d \tau}+\frac{1}{3} \theta^{2} \leq 0
$$

which has a solution in the following form

$$
\frac{1}{\theta(\tau)} \geq \frac{1}{\theta(0)}+\frac{1}{3} \tau
$$

If we assume that $\theta(0)$ is negative, the expansion $\theta(\tau)$ must go to the negative infinity along that geodesic within a proper time

$$
\tau \leq \frac{3}{|\theta(0)|}
$$

whose consequence coincides with that of Hawking and Penrose Hawking \& Penrose (1970).

\section{Singularities in geodesic surface congruence for the null direction}

\subsection{Formalism of null stringy congruence}

Next, we investigate the congruence of the null strings, where the tangent vector of a null curve is normal to itself. See Karlhede \& Lindström (1986); Roshchupkin \& Zheltukhin (1995; 1999); Schild (1977) for the proper definition and propagation of the classical null strings. We consider the evolution of vectors in a $(D-2)$-dimensional subspace of spatial vectors normal to the null tangent vector field $k^{a}=(\partial / \partial \lambda)^{a}$, where $\lambda$ is the affine parameter, and to an auxiliary null vector $l^{a}$ which points in the opposite spatial direction to $k^{a}$, normalized by $l^{a} k_{a}=-1$ Carroll (2004) and is parallel transported, namely, $k^{a} \nabla_{a} l^{b}=0$. The spatial vectors in the $(D-2)$-dimensional subspace are then orthogonal to both $k^{a}$ and $l^{a}$.

We now introduce the metrics $n_{a b}$ defined below and $\bar{h}_{a b}$ defined in (41),

$$
n_{a b}=g_{a b}+k_{a} l_{b}+l_{a} k_{b} .
$$

Similarly to the time-like case, we introduce tensor fields $B_{a b}=\nabla_{b} k_{a}$ and $\bar{B}_{a b}$ in (37) satisfying the identities $B_{a b} k^{a}=\bar{B}_{a b} \zeta^{a}=0$ and $-B_{a b} k^{b}+\bar{B}_{a b} \zeta^{b}=0$. We also define the deviation vector $\eta^{a}=(\partial / \partial \alpha)^{a}$ representing the displacement to an infinitesimally nearby world sheet so that 
we can choose $\lambda, \sigma$, and $\alpha$ as coordinates of the three-dimensional submanifold spanned by the world sheets. We then have the commutator relations $£_{k} \eta^{a}=£_{\zeta} \eta^{a}=£_{k} \zeta^{a}=0$ and $k^{a} \nabla_{a} \eta^{b}-\zeta^{a} \nabla_{a} \eta^{b}=\left(B_{a}^{b}-\bar{B}_{a}^{b}\right) \eta^{a}$.

We decompose $B_{a b}$ into three pieces

$$
B_{a b}=\frac{1}{D-2} \theta n_{a b}+\sigma_{a b}+\omega_{a b}
$$

where the expansion, shear, and twist of the stringy congruence along the affine direction are defined as $\theta=B^{a b} n_{a b}, \sigma_{a b}=B_{(a b)}-\frac{1}{D-2} \theta n_{a b}$ and $\omega_{a b}=B_{[a b]}$. It is noteworthy that even though we have the same notations for $B_{a b}, \theta, \sigma_{a b}$ and $\omega_{a b}$ in (45) and (63), the differences of these notations among the time-like sting cases and null string cases are understood in the context. Similarly, we decompose $\bar{B}_{a b}$ into three parts as in the time-like case.

\subsection{Expansion of null stringy congruence}

Taking the ansatz that the expansion $\bar{\theta}$ is constant along the $\sigma$-direction as in the time-like case, we have another Raychaudhuri type equation

$$
\begin{aligned}
\frac{d \theta}{d \lambda}= & -\frac{1}{D-2} \theta^{2}+\frac{1}{D-1} \bar{\theta}^{2}-\sigma_{a b} \sigma^{a b}+\bar{\sigma}_{a b} \bar{\sigma}^{a b} \\
& +\omega_{a b} \omega^{a b}-\bar{\omega}_{a b} \bar{\omega}^{a b}-R_{a b}\left(k^{a} k^{b}-\zeta^{a} \zeta^{b}\right) .
\end{aligned}
$$

Assuming $\omega_{a b}=\bar{\omega}_{a b}, \sigma_{a} b=\bar{\sigma}_{a} b$ and a stringy strong energy condition for null case $R_{a b}\left(k^{a} k^{b}-\right.$ $\left.\zeta^{a} \zeta^{b}\right) \geq 0$ and exploiting the energy-momentum tensor of the perfect fluid, we reproduce the inequality (57) in the time-like congruence of strings. The Raychaudhuri type equation (64) for the null strings then has a solution in the following form:

$$
\frac{1}{\theta} \geq \frac{1}{\theta_{0}}+\frac{1}{D-2}\left(\lambda-\frac{D-2}{D-1} \int_{0}^{\lambda} \mathrm{d} \lambda\left(\frac{\bar{\theta}}{\bar{\theta}}\right)^{2}\right),
$$

where $\theta_{0}$ is the initial value of $\theta$ at $\lambda=0$. We assume again that $\theta_{0}$ is negative. The inequality (65) then implies that $\theta$ must pass through the singularity within an affine length

$$
\lambda \leq \frac{D-2}{\left|\theta_{0}\right|}+\frac{D-2}{D-1} \int_{0}^{\lambda} \mathrm{d} \lambda\left(\frac{\bar{\theta}}{\theta}\right)^{2} .
$$

In the point particle limit with $\bar{B}_{a b}=\bar{\theta}=\bar{\sigma}_{a b}=\bar{\omega}_{a b}=0$ and $\omega_{a b}=0$, we assume that the strong energy condition $R_{a b} k^{a} k^{b} \geq 0$ is satisfied to yield the second inequality of (58) Carroll (2004); Hawking \& Penrose (1970); Wald (1984). If we assume that the initial value is negative, the expansion $\theta$ must go to the negative infinity along that geodesic within a finite affine length Hawking \& Penrose (1970).

\section{Jacobi fields in geodesic surface congruence}

\subsection{Geodesic surface deviation equation}

In this subsection, we consider a fiber bundle $\pi: M \rightarrow X$ over a spacetime four-manifold $X$ with a Calabi-Yau manifold as a fiber space. Let $M$ be a $n$-dimensional manifold with a metric $g_{a b}$. The action for a string is proportional to the area of the surface spanned in the total space 
$M$ by the evolution along the time direction of the string in $F$. We parametrize the surface generated by a closed string by two world sheet coordinates $\tau$ and $\sigma$, and then we have the corresponding vector fields $\xi^{a}=\left(\frac{\partial}{\partial \tau}\right)^{a}$ and $\zeta^{a}=\left(\frac{\partial}{\partial \sigma}\right)^{a}$. The Nambu-Goto string action is given by Goto (1971); Nambu (1970)

$$
S=-\iint d \tau d \sigma f(\tau, \sigma)
$$

where $0 \leq \tau \leq T$ and $0 \leq \sigma \leq 2 \pi$, and $f(\tau, \sigma)=\left[(\xi, \xi)^{2}-(\xi, \xi)(\zeta, \zeta)\right]^{1 / 2}$.

We perform a variation of the surfaces $\gamma_{a}(\tau, \sigma)$ traced by the closed string during its evaluation to find the geodesic surface equation by the least action principle. Let the vector field $\eta^{a}=\left(\frac{\partial}{\partial \alpha}\right)^{a}$ be the deviation vector field. Since we have gauge degrees of freedom, we can choose the orthonormal gauge $\xi \cdot \zeta=0$ and $\xi \cdot \xi+\zeta \cdot \zeta=0$. The first variation is

$$
\frac{d s}{d \alpha}=\iint d \tau d \sigma \eta_{b}\left(-\xi^{a} \nabla_{a} \xi^{b}+\zeta^{a} \nabla_{a} \zeta^{b}\right)
$$

where we use the endpoint condition $\eta^{a}(0)=\eta^{a}(\tau)=0$ and periodic condition $\eta^{a}(\sigma+2 \pi)=$ $\eta^{a}(\sigma)$. In Cho \& Hong (2007) we have the geodesic surface equation.

$$
-\xi^{a} \nabla_{a} \xi^{b}+\zeta^{a} \nabla_{a} \zeta^{b}=0
$$

When $\left.\frac{d s}{d \alpha}\right|_{\alpha=0}=0$, then the second variation is given by

$$
\left.\frac{d^{2} S}{d \alpha^{2}}\right|_{\alpha=0}=-\iint d \tau d \sigma\left[\frac{d^{2} \eta^{i}}{d \tau^{2}}+\left(R_{\tau j \tau}{ }^{i}-R_{\sigma j \sigma}{ }^{i}\right)\right] \eta_{i} .
$$

We have the geodesic surface deviation equation in Cho \& Hong (2007)

$$
\frac{d^{2} \eta^{i}}{d \tau^{2}}+\left(R_{\tau j \tau}^{i}-R_{\sigma j \sigma}^{i}\right) \eta^{i}=0
$$

In Cho \& Hong (2007), locally $\gamma$ minimizes the Nambu-Goto string action if and only if $\gamma$ is a geodesic surface with no string conjugate to $p=\gamma(0, \sigma)$ between $\gamma(0, \sigma)=p$ and $q=\gamma(\tau, \sigma)$.

\subsection{Singularity in geodesic surface congruence}

In the orthogonal gauge, we introduce two tensor fields :

$$
B_{a b}=\nabla_{b} \xi_{a}, \quad \bar{B}_{a b}=\nabla_{b} \zeta_{a} .
$$

Define the metrics $h_{a b}=g_{a b}+\xi_{a} \xi_{b}$ and $\bar{h}_{a b}=g_{a b}-\zeta_{a} \zeta_{b}$. Let $B_{a b}$ split $B_{a b}$ into three pieces Hawking \& Penrose (1970); Wald (1984)

$$
B_{a b}=\frac{1}{n-1} \theta h_{a b}+\sigma_{a b}+\omega_{a b}
$$


where the expansion, shear, and twist of the stringy congruence along the time direction are defined as $\theta=B^{a b} h_{a b}, \sigma_{a b}=B_{(a b)}-\frac{1}{n-1} \theta h_{a b}$ and $\omega_{a b}=B_{[a, b]}$. Similarly, we split

$$
\bar{B}_{a b}=\frac{1}{n-1} \bar{\theta}, \bar{h}_{a b}+\bar{\sigma}_{a b}+\bar{\omega}_{a b}
$$

where $\bar{\theta}=\bar{B}^{a b} \bar{h}_{a b}, \quad \bar{\sigma}_{a b}=\bar{B}_{(a b)}-\frac{1}{n-1} \bar{\theta} \bar{h}_{a b}$ and $\bar{\omega}_{a b}=\bar{B}_{[a, b]}$.

Taking an ansatz that the expansion $\bar{\theta}$ is constant along the $\sigma$-direction, we have a Raychaudhuri-type equation

$$
\frac{d \theta}{d \tau}=\frac{1}{n-1}\left(\theta^{2}-\bar{\theta}^{2}\right)-\sigma_{a b} \sigma^{a b}+\bar{\sigma}_{a b} \bar{\sigma}^{a b}+\omega_{a b} \omega^{a b}-\bar{\omega}_{a b} \bar{\omega}^{a b}-R_{a b}\left(\xi^{a} \xi^{b}-\zeta^{a} \zeta^{b}\right) .
$$

In Cho \& Hong (2008), assuming that $\omega_{a b}=\bar{\omega}_{a b}, \sigma_{a b}=\bar{\sigma}_{a b}$ and a stringy strong energy condition $R_{a b}\left(\xi^{a} \xi^{b}-\zeta^{a} \zeta^{b}\right) \geq 0$, we have

$$
\frac{1}{\theta} \geq \frac{1}{\theta_{0}}+\frac{1}{n-1}\left(\tau-\int_{0}^{\tau} d \tau\left(\frac{\bar{\theta}}{\theta}\right)^{2}\right),
$$

where $\theta_{0}=\theta(0)$ is the initial value. If $\theta_{0}<0$ so that the congruence is initially converging, then $\theta$ must be pass through the singular point within a proper time

$$
\tau \leq \frac{n-1}{\left|\theta_{0}\right|}+\int_{0}^{\tau} d \tau\left(\frac{\bar{\theta}}{\theta}\right)^{2} .
$$

\subsection{Jacobi fields along geodesic surfaces}

An $n$-dimensional manifold $M$ is said to be conformally symmetric if there is a smooth map $\lambda: M \rightarrow \mathbf{R}$ and for parallel vector fields $U, V$ and $W$ along a curve $\gamma$ is $M$ there is a parallel vector field $X$ along $\gamma$ such that the curvature tensor

$$
R(U, V) W=\lambda X .
$$

For instance, locally symmetric manifolds or symmetric manifolds are conformally symmetric Green et al. (1987); Milnor (1963). From now on we assume that $M$ is conformally symmetric. Let $\gamma_{\alpha}(\tau, \sigma)$ be a variation of geodesic surfaces with the corresponding vector fields $\xi=\frac{\partial}{\partial \tau}, \zeta=\frac{\partial}{\partial \sigma}$ and the deviation vector field $\eta=\frac{\partial}{\partial \alpha}$. Then the geodesic surface deviation equation for the Nambu-Goto string action yields

$$
\frac{d^{2} \eta}{d \tau^{2}}+[R(\xi, \eta) \xi-R(\zeta, \eta) \zeta]=0
$$

where we can construct the variation vectors $\eta(\tau)$ as vectors associated with the centers of the string of the two nearby geodesic surfaces at the time $\tau$. The solution $\eta$ of the geodesic surface deviation equation is called a Jacobi field on the geodesic surface $\gamma_{0}$. Let $\xi_{0}$ be the time direction velocity vector at the string $P=\gamma_{0}(0,0)$, and $\zeta_{0}$ be the string direction velocity vector at the string $\gamma_{0}(0,0)$. We have that the linear transformation

$$
R(\xi, \cdot) \xi-R(\zeta, \cdot) \zeta: T_{p} M \rightarrow T_{p} M
$$


is self-adjoint. Indeed, for any velocity vectors $X$ and $X^{\prime}$ in $T_{p} X$

$$
\left\langle R(\xi, X) \xi-R(\zeta, X) \zeta, X^{\prime}\right\rangle=\left\langle X, R\left(\xi, X^{\prime}\right) \xi-R\left(\zeta, X^{\prime}\right) \zeta\right\rangle .
$$

Let vectors $U_{1}, \cdots, U_{n} \in T_{p} M$ be an orthonormal basis such that

$$
R\left(\xi, U_{i}\right) \xi-R\left(\zeta, U_{i}\right) \zeta=\lambda_{i} U_{i}
$$

where $\lambda_{1}, \cdots, \lambda_{n}$ are the eigenvalues of the self-adjoint operator $R(\xi, \cdot) \xi-R(\zeta, \cdot) \zeta$. Extend the $U_{i}$ to vector fields along $\gamma_{0}$ by parallel translation. Since $M$ is conformally symmetric, there are eigenfunctions $\lambda_{i}: M \rightarrow \mathbf{R}$ such that

$$
R\left(\xi, U_{i}\right) \xi-R\left(\zeta, U_{i}\right) \zeta=\lambda_{i} U_{i}
$$

along the geodesic surface $\gamma_{0}$. The Jacobi field along the geodesic surface $\gamma_{0}$ may be expressed uniquely as

$$
\eta(\tau)=\eta_{1}(\tau) U_{1}(\tau)+\cdots \eta_{n}(\tau) U_{n}(\tau) .
$$

Then the geodesic surface deviation equation takes the form

$$
\sum_{i=1}^{n}\left[\frac{d^{2} \eta_{i}(\tau)}{d \tau^{2}}+\lambda_{i}(\tau) \eta_{i}(\tau)\right] U_{i}(\tau)=0
$$

Since the $U_{i}$ are every where linearly independent, the equation is equivalent to the system of $n$ equations

$$
\frac{d^{2} \eta_{i}(\tau)}{d \tau^{2}}+\lambda_{i}(\tau) \eta_{i}(\tau)=0, \quad i=1, \cdots, n
$$

Suppose that $\tau=0$ is the singular point of the expansion $\theta$ so that the congruence of geodesic surfaces is converging, and that the expansion is constant along the $\sigma$-direction. Assume that $\lambda_{i}(\tau)=\frac{1}{\tau}$, and $\eta_{i}(\tau):=c_{i 0}+c_{i 1} \tau+c_{i 2} \tau^{2}+\cdots$. Then the system of $n$ geodesic surface deviation equations

$$
\frac{d^{2} \eta_{i}(\tau)}{d \tau^{2}}+\lambda_{i}(\tau) \eta_{i}(\tau)=0
$$

By the initial condition $\eta(0)=0, \eta_{i}(0)=0=c_{i 0}$ for all $i$. By the differentiation of $\eta_{i}(\tau)$ with respect to $\tau$, we have

$$
\begin{aligned}
\frac{d \eta_{i}(\tau)}{d \tau} & =c_{i 1}+2 c_{i 2} \tau+3 c_{i 3} \tau^{2}+\cdots+n c_{i n} \tau^{n-1}+\cdots \\
\frac{d^{2} \eta_{i}(\tau)}{d \tau} & =(2 \cdot 1) c_{i 2}+(3 \cdot 2) c_{i 3} \tau^{1}+\cdots+(n \cdot(n-1)) c_{i n} \tau^{n-2}+\cdots
\end{aligned}
$$

By multiplying $\lambda_{i}(\tau)$ to $\eta_{i}(\tau)$,

$$
\lambda_{i}(\tau) \eta_{i}(\tau)=c_{i 1}+c_{i 2} \tau^{1}+\cdots+c_{i(n-1)} \tau^{n-2}+\cdots .
$$

Thus,

$$
(2 \cdot 1) c_{i 2}=-c_{i 1}, \quad(3 \cdot 2) c_{i 3}=-c_{i 2}, \quad \cdots, \quad(n \cdot(n-1)) c_{i n}=-c_{i(n-1)} .
$$


Then by induction we have

$$
c_{i n}=-\frac{1}{n(n-1)} c_{i(n-1)}=(-1)^{n-1} \frac{1}{n !(n-1) !} c_{i 1} .
$$

Let $c_{i 1}=c_{i}$. Then the solution

$$
\eta_{i}(\tau)=c_{i} \sum_{n=1}^{\infty}(-1)^{n-1} \frac{1}{n !(n-1) !} \tau^{n}, \quad i=1, \cdots, n,
$$

where $c_{i}$ is a constant, $i=1, \cdots, n$.

Suppose that the singularity exists in geodesic surface congruences of the locally symmetric, spacetime manifold. Then the Jacobi field along geodesic surface $\gamma_{0}$ in the geodesic surface congruence is given by

$$
\eta(\tau)=\sum_{i=1}^{n} \eta_{i}(\tau) U_{i}(\tau)
$$

where $\quad \eta_{i}(\tau)=c_{i} \sum_{k=1}^{\infty}(-1)^{k-1} \frac{\tau^{k}}{k !(k-1) !}$, and $c_{i}$ is constant, $i=1, \cdots, n$.

\subsection{Observations}

If $M$ is a symmetric manifold and if $\xi$ and $\zeta$ are parallel, the Jacobi equation becomes

$$
\frac{d^{2} \eta_{i}(\tau)}{d \tau^{2}}+\lambda_{i}(\tau) \eta_{i}(\tau)=0
$$

where the eigenfunctions $\lambda_{i}(\tau)$ of the operator $R(\xi, \cdot) \xi-R(\zeta, \cdot) \zeta$ are independent of $\tau$.

$$
\begin{aligned}
& \text { If } \lambda_{i}>0 \text {, then } \eta_{i}(\tau)=c_{i} \sin \left(\sqrt{\lambda_{i}} \tau\right) . \\
& \text { If } \lambda_{i}=0 \text {, then } \eta_{i}(\tau)=c_{i} \tau \text {. } \\
& \text { If } \lambda_{i}<0 \text {, then } \eta_{i}(\tau)=c_{i} \sinh \left(\sqrt{\left|\lambda_{i}\right|} \tau\right) .
\end{aligned}
$$

Thus if $\lambda_{i} \leq 0, \eta_{i}(\tau)$ does not vanish except $\tau=0$.

In our assumption, the eigenfunction $\lambda_{i}(\tau)=\frac{1}{\tau}$ is a kind of curvature at time $\tau$. If $\tau \rightarrow \infty$ then $\lambda_{i}(\tau) \rightarrow 0$, since the universe is expanding by observation. If $\tau \rightarrow 0$, then $\lambda_{i}(\tau) \rightarrow \infty$, since the point $\tau=0$ is the singular point which the universe comes into being by Big Bang. The sum

$$
\eta_{i}(\tau)=c_{i} \sum_{k=1}^{\infty}(-1)^{k-1} \frac{1}{k !(k-1) !} \tau^{k}
$$

converges absolutely for all $\tau$. Indeed, by the ratio test

$$
\lim _{k \rightarrow \infty}\left|\frac{(-1)^{k} \frac{1}{(k+1) ! k !} \tau^{k+1}}{(-1)^{k-1} \frac{1}{k !(k-1) !} \tau^{k}}\right|=\lim _{k \rightarrow \infty} \frac{|\tau|}{(k+1) k}=0<1, \text { for all ø. }
$$

We may think topologically our manifold $M \simeq[0, \tau] \times D^{3}(\tau) \times X \simeq D^{4}(\tau) \times X$ where $\tau$ is the time and $X$ is the $C Y$-manifold which is hidden. $M$ is compact and we live in $\partial D^{4}(\tau)=S^{3}(\tau)$ which is our visible universe at time $\tau$. 
If $\lambda_{i}(\tau)=\frac{a_{i}}{\tau}$ for some constant $a_{i}$ along $U_{i}$ direction, then the solutions of the Jacobi equations are

$$
\eta_{i}(\tau)=c_{i} \sum_{n \geq 1}(-1)^{n-1} \frac{a_{i}^{n-1}}{n !(n-1) !} \tau^{n}, i=1, \cdots, n .
$$

The series of the right hand have the radius of convergence $\infty$.

\section{Conclusions}

We consider phase transition which is assumed to have happened in the evolution of the universe around 75,000 years after the Big Bang. Assuming that the early universe was filled with a perfect fluid consisting of massive particles and/or massless particles and using the strong energy condition, one could find equations of state for each particle.

Specifically, the equation of state of the massive particle is different from that of the massless particle, which indicates that the massive particle phase is not the same as the massless particle one. In the standard cosmology based on the Hawking-Penrose singularity theorem and inflationary scenario, it is believed that, after the Big Bang explosion, a massless particle (or radiation) dominated phase occurred followed by a massive particle (or matter) dominated one, even though there was a hot thermalization period of radiation and matter immediately after the Big Bang. Moreover, as in the phase transition in water, a phase transition exists between massive particle and massless particle phases in the universe.

In the above explanations of the Hawking-Penrose singularity theorem and the ensuing standard cosmology, they assumed that massive and massless particles are point-like. Now let us discuss stringy cosmology which has been developed recently Cho \& Hong (2007) and is based on the string theory Polchinski (1999). The string theory has given us a better understanding of the universe and has provided an analytical tool for studying the nature of the universe. It was proposed in the string theory that massive and massless particles in the universe can be described by vibrating strings instead of point particles. As in the standard (point particle) cosmology, using the strong energy condition modified by stringy corrections, one can find the stringy singularity theorem, which is similar to the Hawking-Penrose singularity theorem except for the fact that evolution to a singularity occurs at different spacetime Cho \& Hong (2007).

Exploiting the stringy strong energy condition together with the assumption that the early universe was occupied with a perfect fluid of massive stringy particles and/or massless stringy particles, one obtains equations of state for each stringy particle. Surprisingly, the massive stringy particles and massless stringy particles produce the same equation of state. This implies that both massive stringy particles and massless stringy particles are in the same phase and thus there is no phase transition in the stringy universe Cho \& Hong (2007). Here, we emphasize that the equations of state obtained from the standard and stringy cosmologies govern the evolution of the universe, since these equations originate from the initial conditions of the (stringy) singularity theorems. Immediately after the Big Bang explosion, in the stringy cosmology, the massless stringy particle dominated phase and the massive stringy particle dominated phase took place simultaneously. In the stringy universe, a massless-massive stringy particle mixture state without any phase transitions exists. These features in the stringy universe are drastically different from those in the standard point particle cosmology. In fact, these differences originate from the lone fact that the particles are composed of strings instead of points. 
In the standard cosmology, after the radiation and matter dominated phases a dark energy dominated phase exists. In this inflationary scenario, a sequence of epoches occurred: Big Bang, radiation dominated phase, matter dominated phase, and dark energy dominated phase. In brane cyclic cosmology, which is also based on the string (or D brane) theory, the universe is assumed to be cyclic in evolution: Big Bang, radiation dominated phase, matter dominated phase, dark energy dominated phase, Big Crunch, and again Big Bang Steinhardt \& Turok (2002). Moreover, both in the standard and stringy cosmologies exploiting the (stringy) singularity theorems, there exists an initial state with a negative expansion rate and the ensuing evolution to the singularity. One can thus claim that the stringy cosmology is also cyclic, similar to the brane cyclic cosmology, but modified: Big Bang, radiation-matter mixture phase, dark energy dominated phase, Big Crunch, and again Big Bang, which is consistent with the fact that both the stringy and brane cyclic cosmologies share the same characteristics that these scenarios use the string features.

We want to detect the so-called quark-gluon plasma state, which is assumed to exist in an extremely hot soup of quarks and gluons. Both in the standard and stringy cosmologies, this quark-gluon plasma state is supposed to occur immediately after the Big Bang of the tiny early universe manufactured in the Large Hadron Collider. However, there are drastically different ensuing processes in these two scenarios. Namely, in the standard cosmology, the quark-gluon plasma state can exist shortly and disappear eventually to enter the radiation dominated phase, while in the stringy cosmology the quark-gluon plasma state can develop into particles such as protons and neutrons and sustain the radiation and matter mixture phase. We recall that as far as radiation and matter are concerned, the mixture of these two coexists in the present universe. It is expected that the Large Hadron Collider will be able to detect the procedure of particle states along with the evolution of the tiny universe planned to occur at the Large Hadron Collider and it will be able to determine which cosmology is viable. More details are reported in Cho \& Hong (2010).

\section{References}

Carroll, S.M. (2004). An Introduction to General Relativity: Spacetime and Geometry, Addition Wesley, New York.

Cho, Y.S. \& Hong, S.T. (2007). Stringy Jacobi fields in Morse theory, Phys. Rev. D 75: 127902.

Cho, Y.S. \& Hong, S.T. (2008). Singularities in geodesic surface congruence, Phys. Rev. D 78: 067301.

Cho, Y.S. (2010). Jacobi fields in geodesic surface congruences, Int. J. Geom. Meth. Mod. Phys. 7: 1407-1412.

Cho, Y.S. \& Hong, S.T. (2010). String reveals secrets of universe, arXiv:1010.3485.

Goto, T. (1971). Relativistic quantum mechanics of one-dimensional mechanical continuum and subsidiary condition of dual resonance model, Prog. Theor. Phys. 46: 1560-1569.

Green, M.B., Schwarz, J.H. \& Witten, E. (1987). Superstring Theory, Cambridge Univ. Press, Cambridge.

Hawking, S.W. \& Penrose, R. (1970). The Singularities of gravitational collapse and cosmology, Proc. Roy. Soc. Lond. A 314: 529-548.

Karlhede, A. \& Lindström, U. (1986). The classical bosonic string in the zero tension limit, Class. Quantum Grav. 3: L73-L75.

Milnor, J. (1963). Morse Theory. Princeton University Press, Princeton. 
Misner, C.W., Thorne K.P. \& Wheeler, J.A. (1972). Gravitation, Freeman, San Francisco.

Nambu, Y. (1970). Lecture at the Copenhagen Symposium.

Polchinski, J. (1999). String Theory, Cambridge Univ. Press, Cambridge.

Roshchupkin, S.N. \& Zheltukhin, A. A. (1995). Friedmann universes and exact solutions in string cosmology, Class. Quantum Grav. 12: 2519-2524;

Roshchupkin, S.N. \& Zheltukhin, A. A. (1999). Variational principle and a perturbative solution of nonlinear string equations in curved space, Nucl. Phys. B 543: 365-386.

Scherk, J. (1975). An introduction to the theory of dual models and strings, Rev. Mod. Phys. 47: 123-164.

Schild, A. (1977). Classical null strings, Phys. Rev. D 16: 1722-1726.

Steinhardt, P.J. \& Turok, N. (2002). A cyclic model of the universe, Science 296: 1436-1439.

Wald, R.M. (1984). General Relativity, University of Chicago Press, Chicago. 


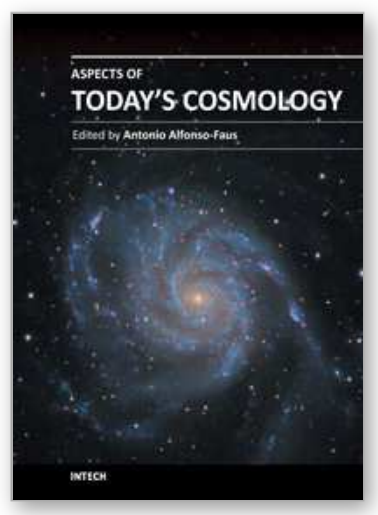

\author{
Aspects of Today's Cosmology \\ Edited by Prof. Antonio Alfonso-Faus
}

ISBN 978-953-307-626-3

Hard cover, 396 pages

Publisher InTech

Published online 09, September, 2011

Published in print edition September, 2011

This book presents some aspects of the cosmological scientific odyssey that started last century. The chapters vary with different particular works, giving a versatile picture. It is the result of the work of many scientists in the field of cosmology, in accordance with their expertise and particular interests. Is a collection of different research papers produced by important scientists in the field of cosmology. A sample of the great deal of efforts made by the scientific community, trying to understand our universe. And it has many challenging subjects, like the possible doomsday to be confirmed by the next decade of experimentation. May be we are now half way in the life of the universe. Many more challenging subjects are not present here: they will be the result of further future work. Among them, we have the possibility of cyclic universes, and the evidence for the existence of a previous universe.

\title{
How to reference
}

In order to correctly reference this scholarly work, feel free to copy and paste the following:

Yong Seung Cho and Soon-Tae Hong (2011). Singularities and Thermodynamics of Geodesic Surface Congruences, Aspects of Today's Cosmology, Prof. Antonio Alfonso-Faus (Ed.), ISBN: 978-953-307-626-3, InTech, Available from: http://www.intechopen.com/books/aspects-of-today-s-cosmology/singularities-andthermodynamics-of-geodesic-surface-congruences

\section{INTECH}

open science | open minds

\author{
InTech Europe \\ University Campus STeP Ri \\ Slavka Krautzeka 83/A \\ 51000 Rijeka, Croatia \\ Phone: +385 (51) 770447 \\ Fax: +385 (51) 686166 \\ www.intechopen.com
}

\author{
InTech China \\ Unit 405, Office Block, Hotel Equatorial Shanghai \\ No.65, Yan An Road (West), Shanghai, 200040, China \\ 中国上海市延安西路65号上海国际贵都大饭店办公楼 405 单元 \\ Phone: +86-21-62489820 \\ Fax: +86-21-62489821
}


(C) 2011 The Author(s). Licensee IntechOpen. This chapter is distributed under the terms of the Creative Commons Attribution-NonCommercialShareAlike-3.0 License, which permits use, distribution and reproduction for non-commercial purposes, provided the original is properly cited and derivative works building on this content are distributed under the same license. 